\title{
Multiple Sclerosis Disease-Modifying Therapy Prescribing Patterns in Ontario
}

\author{
James J. Marriott, Muhammad Mamdani, Gustavo Saposnik, Tara Gomes, \\ Michael Manno, Paul W. O’Connor
}

\begin{abstract}
Background: Differences in Multiple sclerosis (MS) disease-modifying therapy (DMT) prescribing patterns between different groups of neurologists have not been explored. Objective: To examine concentrations of prescribing patterns and to assess if MS-specialists use a broader range of DMTs relative to general neurologists. Methods: We conducted a cross-sectional study using administrative claims databases in Ontario, Canada to link neurologists to 2009 DMT prescription data. MS specialization was defined using both practice location and prescription patterns. Lorenz curves and Gini coefficients were constructed to examine prescribing patterns, separating neurologist characteristics dichotomously and separating Avonex from the other standard DMTs (Betaseron, Rebif and Copaxone). Gini coefficient $95 \%$ confidence intervals (CIs) were derived using jack-knife statistical techniques. Results: Prescriptions were highly concentrated with $12 \%$ of Ontario neurologists prescribing $80 \%$ of DMTs. There was a trend towards Avonex being more commonly prescribed relative to the other DMTs. When MS specialization was defined by DMT prescribing, high-volume prescribing neurologists showed a broader range of DMT prescribing (Gini 0.38-0.44) in comparison to low-volume prescribers (Gini 0.57-0.66). Conclusions: The majority of DMTs are prescribed by a small subset of neurologists. High-volume prescribing MSspecialists show more variability in DMT use while low-volume prescribers tend to individually focus on a narrower range of DMTs.
\end{abstract}

RÉSUMÉ: Mode de prescription des traitements modificateurs de la maladie dans la sclérose en plaques en Ontario. Contexte : Les différences dans le mode de prescription des traitements modificateurs de la maladie (TMM) dans la sclérose en plaques (SP) entre différents groupes de neurologues n'ont jamais été étudiées. Objectif : Le but de l'étude était d'examiner les concentrations de modes de prescription et d'évaluer si les spécialistes de la SP utilisent un éventail plus large de TMM que les neurologues généralistes. Méthodes : Nous avons effectué une étude transversale des données de réclamations administratives dans les bases de données de l'Ontario, au Canada, pour relier les neurologues aux données de prescription de TMM en 2009. La spécialisation en SP était définie au moyen du lieu de pratique et du mode de prescription. Nous avons établi des courbes de Lorenz et des coefficients de Gini pour examiner les modes de prescription, séparant les caractéristiques des neurologues de façon dichotomique et séparant l'Avonex des autres TMM standards (Betaseron, Rebif et Copaxone). Les intervalles de confiance (IC) à 95\% du coefficient Gini ont été calculés au moyen de la méthode de rééchantillonnage « jack-knife ». Résultats : Les prescriptions étaient fortement concentrées, soit $12 \%$ des neurologues de l'Ontario qui prescrivaient $80 \%$ des TMM. La prescription d'Avonex était légèrement plus fréquente que la prescription des autres TMM. Si la spécialisation en SP était définie par la prescription de TMM, les neurologues qui en prescrivaient beaucoup prescrivaient des TMM plus variés (Gini 0,38 à 0,44 ) que ceux qui n'en prescrivaient pas beaucoup (Gini 0,57 à 0,66 ). Conclusions : La majorité des TMM sont prescrits par un petit sous-groupe de neurologues. Les spécialistes en SP qui en prescrivent beaucoup prescrivent des TMM plus variés alors que ceux qui en prescrivent peu ont tendance à avoir leurs préférences individuelles et à prescrire une gamme de produits plus restreinte.

Can J Neurol Sci. 2013; 40: 67-72

The four disease-modifying therapies (DMTs) introduced in the late 1990s, Avonex, ${ }^{1}$ Betaseron, ${ }^{2-4}$ Rebif $^{5}$ and Copaxone, ${ }^{6}$ are all partially effective in the treatment of relapsing forms of multiple sclerosis (MS). In the pivotal trials, relapse rates were decreased $18 \%$ by Avonex and $30 \%$ by the other three agents, with variable effects on sustained Expanded Disability Status Scale (EDSS) progression and magnetic resonance imaging (MRI) activity. ${ }^{1-6}$ Head-to-head clinical trials comparing Copaxone with either Rebif or Betaseron did not demonstrate a clinical advantage between any of these drugs, ${ }^{7-8}$ however individual patients may certainly respond better to one medication over another. ${ }^{9}$ Further, given the modest treatment efficacy of all agents and their considerable expense, there is ongoing debate about the cost-effectiveness of DMTs. ${ }^{10}$ In addition to the issue of high cost, the four first-line DMTs are all injectable medications with both local injection-site and rare systemic side-effects including lymphopenia and liver toxicity with the interferons and idiosyncratic post-injection reactions with Copaxone.

Multiple sclerosis is treated by a wide spectrum of neurologists ranging from community-based generalist

From the Department of Internal Medicine (Neurology), University of Manitoba (JJM), Winnipeg, Manitoba; Department of Health Policy (MuM, GS), Management, and Evaluation; Leslie Dan Faculty of Pharmacy (MuM, TG), Department of Medicine (Neurology), University of Toronto (GS, PWO); Institute of Clinical Evaluative Sciences (MuM, GS, TG); Stroke Research Unit (GS), Mobility Program; Multiple Sclerosis Clinic and MS Research and Evoked Potentials Laboratory (PWO), Keenan Research Centre of the Li Ka Shing Knowledge Institute of St. Michael's Hospital (MuM, GS, PWO), St. Michael's Hospital; Ontario HIV Treatment Network (MiM), Toronto, Canada.

Received May 22, 2012. Final Revisions Submitted July 19, 2012. Correspondence to: James J Marriott, Section of Neurology, Health Sciences Centre, GF543-820 Sherbrook Street, Winnipeg, Manitoba, R3A 1R9, Canada.

Email: jmarriott@hsc.mb.ca. 
neurologists to academic MS-specialists who are affiliated with tertiary hospital based MS Clinics. Differences in DMT prescribing patterns between different groups of neurologists have not been previously explored. Given the ongoing question of cost-effectiveness of the first line injectable DMTs, it would be useful to know if prescribing practices vary by prescribercharacteristics. Anecdotal observation at the St. Michael's Hospital MS Clinic (Toronto, Ontario) suggested that: (1) neurologists frequently sent patients to the clinic prior to the initiation of DMT patients, (2) those patients seen in the clinic after starting DMT in the community tended to be on Avonex. As a single weekly injection, Avonex might be perceived as less onerous for patients and easier to monitor in the community.

This project was therefore designed to assess the distribution of DMT prescriptions among neurologists and to analyze if prescription patterns varied between specialist and generalist neurologists. It was hypothesized that a minority of neurologists generate the majority of DMT prescriptions with an overrepresentation of Avonex prescriptions amongst the cohort of DMT-prescribing neurologists. It was further hypothesized that MS specialists (defined using either proximity to an MS Clinic or DMT prescription volume) would demonstrate a broader range of DMT prescriptions while there would be a preferential trend towards Avonex prescriptions amongst community neurologists.

\section{Materials AND Methods}

Disease-modifying therapies prescribing in Ontario, Canada in 2009 were assessed in a cross-sectional study using population-based administrative claims databases. All data was collected from the linked, anonymized public health services databases for the province of Ontario maintained at the Institute for Clinical Evaluative Sciences (ICES). This project was approved by the Research Ethics Board of Sunnybrook Health Sciences Centre, Toronto, Ontario.

Prescription claim data stored in the Ontario Drug Benefit (ODB) program database includes all prescriptions dispensed to Ontario residents aged 65 years or older, on social assistance, or receiving partial/complete coverage through the Ontario catastrophic drug cost assistance program. Patients who self-pay or have full private drug coverage are not captured by the ODB, and thus are not included in this study.

All DMT prescriptions obtained by ODB recipients between January 1st, 2009 and December 31st, 2009 inclusive were obtained from the ODB claim database. Prior to being added to the provincial formulary in September 2009, Tysabri was only available through private drug plans, so this analysis was restricted to the four first-line DMTs. Each DMT script was linked to the corresponding prescribing physician listed in the ICES Physician Database (IPDB). All physicians not classified as actively practising neurologists and all individual prescriptions that could not be linked to a prescribing physician were excluded from the analysis.

Neurologist specialization was defined in two ways. First, all Ontario neurologists were characterized by whether or not they worked in a geographical area corresponding to an MS Clinic. This was done by querying the IPDB to see whether each physician's practice location forward sortation area (FSA), the first three alphanumeric characters of the postal code, corresponded to any of the eight hospital-based Ontario MS clinics (as listed on http://mssociety.ca/en/help/clinics.htm\# ontario, accessed February 3, 2010). Forward sortation areas are the standard geographical unit used in ICES analyses and in this context, served to approximate a geographical region small enough to localize a hospital-based MS clinic. Specialization was also indirectly examined using the number of DMT prescriptions prescribed by a physician as a surrogate for MS sub-speciality interest. An arbitrary decision was made to dichotomize Ontario neurologists as MS specialists if they prescribed greater than one hundred DMT prescriptions in 2009.

Lorenz curves and Gini coefficients were constructed to examine the distribution of DMT prescribing in Ontario. Lorenz curves were developed to study the wealth distributions of populations and have been adapted to other disciplines, including drug utilization where the cumulative percentages of patients or prescribing physicians is plotted against the

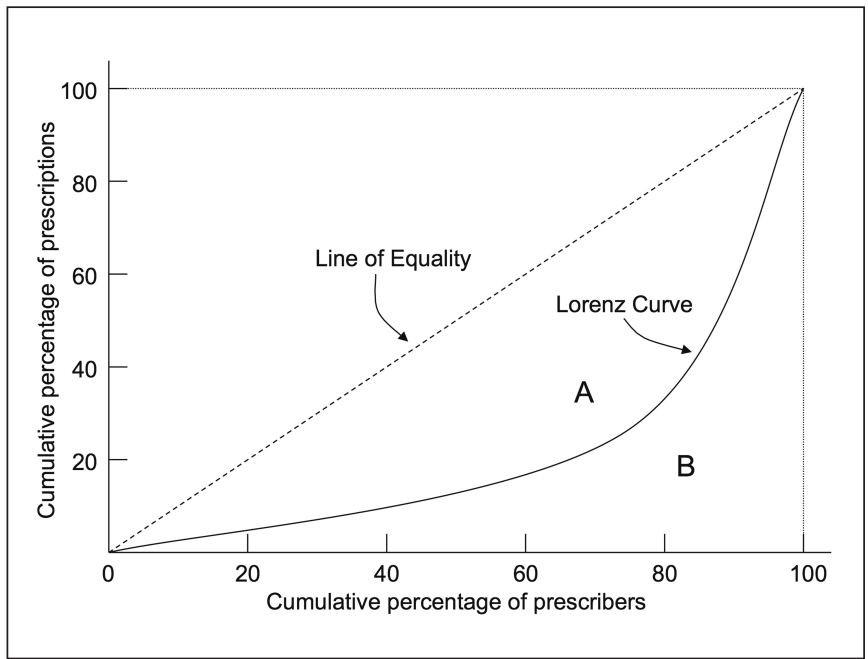

Figure 1: Sample Lorenz curve

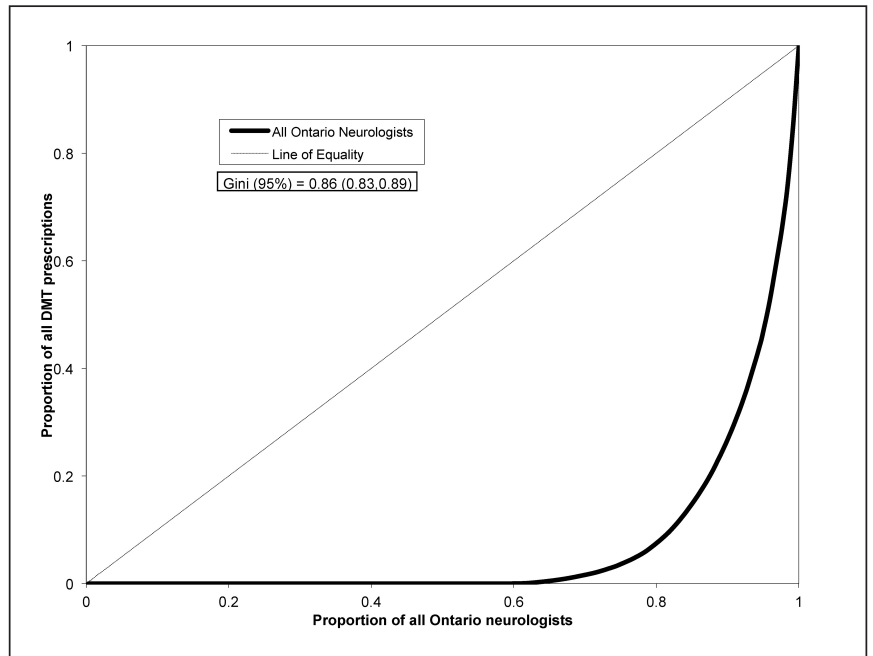

Figure 2: DMT prescribing of all Ontario neurologists 
Table 1: Disease-modifying therapy (DMT) prescriptions stratified by characteristics of prescribing neurologist

\begin{tabular}{|c|c|c|c|c|c|c|}
\hline \multirow{2}{*}{$\begin{array}{l}\text { Neurologist Characteristic } \\
\text { (Number) }\end{array}$} & \multicolumn{6}{|c|}{ DMT Type $(\%)$} \\
\hline & Avonex & Betaseron & Rebif & Copaxone & All Non-Avonex & Any DMT \\
\hline MS Clinic (100) & $1480(21.93)$ & $1345(19.93)$ & $2039(30.21)$ & $1885(27.93)$ & $5269(78.07)$ & $6749(100)$ \\
\hline Non-MS Clinic (186) & $1824(31.03)$ & $973(16.55)$ & $1350(22.96)$ & $1732(29.46)$ & $4055(68.97)$ & $5879(100)$ \\
\hline$\leq 100$ prescriptions (249) & $954(39.8)$ & $386(16.1)$ & $609(25.4)$ & $447(18.7)$ & $1442(60.2)$ & $2396(100)$ \\
\hline$>100$ prescriptions (37) & $2350(23.0)$ & $1932(18.9)$ & $2780(27.2)$ & $3170(31.0)$ & $7882(77.0)$ & $10232(100)$ \\
\hline All (286) & $3304(26.16)$ & $2318(18.36)$ & $3389(26.84)$ & 3617 (28.64) & $9324(73.84)$ & $12628(100)$ \\
\hline
\end{tabular}

cumulative number of prescriptions (Figure 1). ${ }^{11-14}$ A perfectly equal distribution within a population would fall on the "line of equality" bisecting the graph. The Gini coefficient (range 0-1) is defined as the space between the Lorenz curve and line of equality. The Gini coefficients derived from the Lorenz curves were used to compare how unequally DMTs were prescribed by different populations of Ontario neurologists. Standard errors and $95 \%$ confidence intervals (CIs) for the Gini coefficients were calculated using the jackknifing technique of Ogwang. ${ }^{15}$

Four separate analyses were performed, and Lorenz curves were constructed for each. First, the cumulative percentage of all Ontario neurologists was plotted against the cumulative percentage of total DMTs prescribed (ie: all four DMTs were pooled). Next, the cumulative percentage of prescribing neurologists was plotted against the cumulative percentage of prescriptions with each DMT examined individually.

The interaction between MS-specialization and DMT prescribing was examined in the final two analyses. The cumulative percentage of prescribing neurologists was first plotted against the cumulative percentage of DMTs prescribed, stratifying neurologists into those with MS clinic or non-MS

Table 2: Percentages of all Ontario neurologists prescribing specific percentages of DMTs

\begin{tabular}{c|c|c|c}
\hline \multirow{2}{*}{ DMT } & \multicolumn{3}{|c}{ Percentage of DMT prescriptions } \\
\cline { 2 - 4 } & $50 \%$ & $80 \%$ & $100 \%$ \\
\hline Avonex & $5 \%$ & $13 \%$ & $29.02 \%$ \\
\hline Betaseron & $3.5 \%$ & $10 \%$ & $24.13 \%$ \\
\hline Rebif & $3 \%$ & $9 \%$ & $26.22 \%$ \\
\hline Copaxone & $4.5 \%$ & $9 \%$ & $25.52 \%$ \\
\hline Total & $5 \%$ & $12 \%$ & $40.56 \%$ \\
\hline
\end{tabular}

clinic based practice locations. Secondly, this analysis was repeated but with neurologists dichotomized into either high $(>100)$ or low $(\leq 100)$ volume prescribers. In each of these two analyses, the prescriptions were separated into two groups to allow direct comparisons between Avonex and the other DMTs. The first group consisted of Avonex prescriptions alone and the second consisted of all prescriptions for the other three DMTs combined together in aggregate.

\section{RESUlts}

A total of 16790 DMT prescriptions were identified in the ODB database. A total of 2268 scripts could not be linked to a specific Ontario physician due to coding/data entry errors. A further 1894 scripts were not prescribed by a neurologist; $79 \%$ of these were from general practitioners. This left a total of 12628 DMTs prescribed by the 286 neurologists practicing in Ontario in 2009 (Table 1). Disease-modifying therapies prescribing was markedly skewed as only 116 neurologists (41\%) prescribed any DMTs (Table 2). Only 37 (13\%) neurologists were classified as high volume $(>100)$ prescribers,

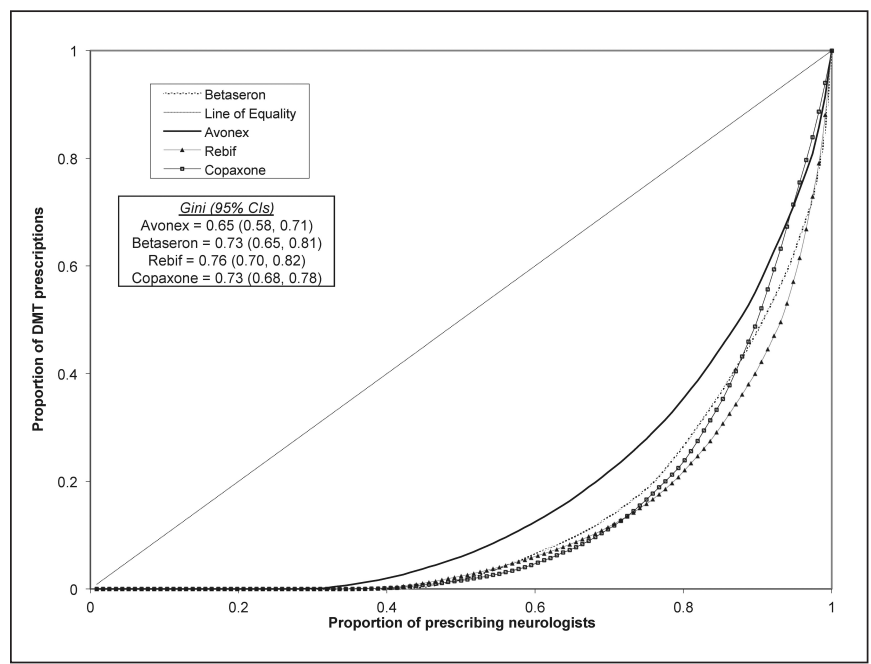

Figure 3: Individual DMT prescribing of all prescribing neurologists 
and $100(35 \%)$ neurologists worked at a site with an MS Clinic. For each of the DMTs, approximately $85 \%$ of the prescriptions were for standard one month/30 day periods.

The Lorenz curve shown in Figure 2 depicts the cumulative percentage of DMT prescriptions dispensed in the province of Ontario in 2009 as a function of the cumulative percentage of neurologists practising in the province. The Gini coefficient of 0.86 (95\% CI: $0.83,0.89)$ reflects the significant skew away from the line of equality that is apparent on visual inspection of the figure. Figure 2 and Table 2 show that $80 \%$ of DMT prescriptions were generated by only $12 \%$ of the neurologists in the province. This percentage was not significantly different when each DMT was examined individually (range $9-13 \%$ for the four DMTs). The final column of Table 2 shows the focus of DMT prescribing to be even more concentrated when each DMT is examined individually (24-29\% for each DMT versus $41 \%$ for all DMTs in aggregate).

Visual inspection of the Lorenz curves for individual DMTs among the cohort of 116 prescribing neurologists indicates that Avonex was the most evenly prescribed. This difference however was not statistically significant as shown by the overlapping Gini coefficient (range 0.65-0.76) 95\% CIs. Even among prescribing neurologists, the distribution of prescribing was markedly unequal. Approximately $10 \%$ of this cohort of neurologists generated $50 \%$ of the DMT prescriptions (Figure 3).

Figure 4 shows the prescribing patterns for MS clinic and non-MS clinic neurologists. As is evident on visual inspection of the overlying curves and confirmed by the overlapping Gini coefficients, no differences were identified between MS specialists (as defined using postal code overlap with an MS clinic) and generalist neurologists .

Figure 5, in contrast shows significant differences in prescribing patterns between specialists and generalist neurologists when high-prescription volume was used as a surrogacy for specialization. The four curve-to-curve

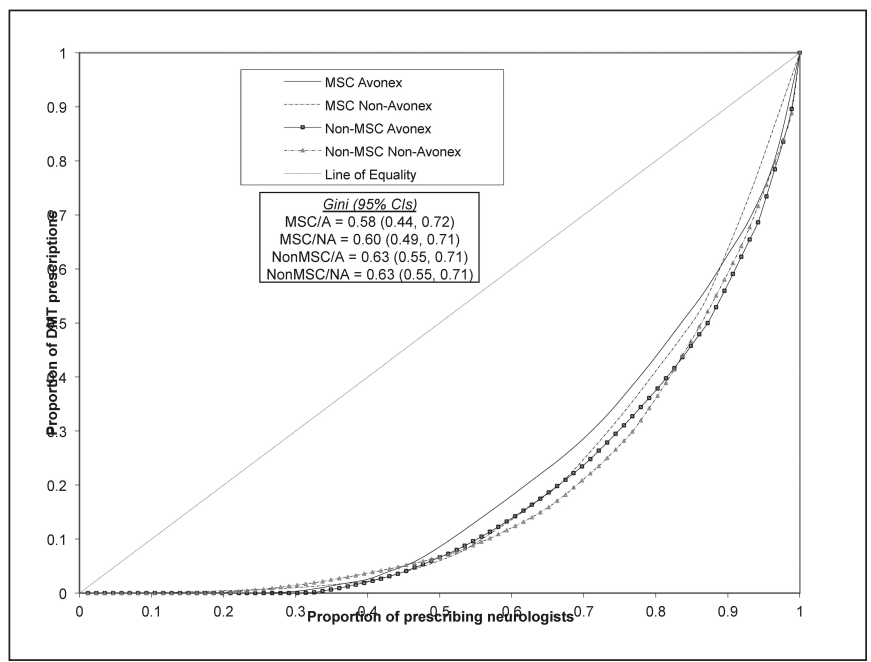

Figure 4: DMT prescribing patterns by specialization. MS Clinic-based and non-MS Clinic-based neurologists have been dichotomized. Avonex prescriptions have been dichotomized from non-Avonex (Betaseron, Rebif, Copaxone) prescriptions. comparisons between the low- and high-volume prescribers were significantly different with non-overlapping Gini coefficient $95 \%$ CIs. One exception to this was the $\leq 100$ prescription/non-Avonex and $>100$ prescription/Avonex curves which did cross (Figure 5). Conversely, the two curve-to-curve comparisons within the low-volume and high-volume groups of prescribers were not significantly different. Both the Avonex and non-Avonex curves for the high-volume prescribers were closer to the line of equality than the two curves for the low-volume prescribing neurologists indicating that the distribution of medications was more even amongst the high-volume prescribers.

\section{Discussion}

This study demonstrates that DMT prescribing is not uniform across the province with only $12 \%$ of Ontario neurologists responsible for $80 \%$ of the DMT prescriptions dispensed through the ODB in 2009. This percentage was approximately the same as those for each of the four DMTs when they were analyzed individually. Even within the cohort of 116 neurologists (40\% of the total number in the province) who treated MS patients with DMTs, roughly $10 \%$ of this cohort of neurologists generated $50 \%$ of the DMT prescriptions. When only those neurologists who prescribed any DMTs were examined in isolation, there was a non-significant trend towards Avonex being the most evenly prescribed DMT.

Divergent results were obtained for the two definitions of MS specialization. The MS-clinic-based definition identified 100 neurologists. Contrary to expectations, no difference was seen between the four Lorenz curves plotted for the MS clinic and non-MS clinic neurologists. Moderate skewing was present with $\sim 20 \%$ of neurologists generating $\sim 60 \%$ of the prescriptions in each group. In contrast, when specialization was defined by prescription volume, the 37 high-volume prescribing neurologists that were isolated were found to prescribe

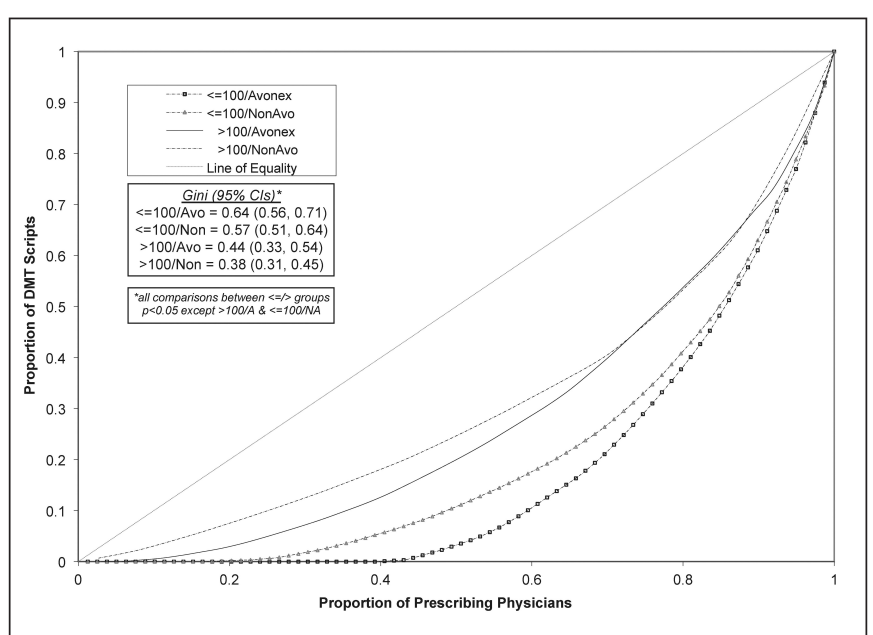

Figure 5: DMT prescribing patterns by specialization. Low-volume ( $\leq 100$ prescriptions in 2009) and high-volume (>100 prescriptions in 2009) neurologists have been dichotomized. Avonex prescriptions have been dichotomized from non-Avonex (Betaseron, Rebif, Copaxone) prescriptions. 
differently from low-volume prescribers. For both the Avonex and non-Avonex DMT groupings, the degree of skew was markedly less with the high-volume prescribers, indicating a more even distribution of prescriptions relative to the lowvolume prescribing neurologists.

If most scripts coming from a postal code corresponding to an MS clinic can be assumed to emanate from an MS-specialist, why are Figures 4 and 5 not more alike? An examination of Table 1 demonstrates that the two surrogates used to define MS specialists are identifying different subsets of Ontario neurologists. The 37/286 (13\%) high-volume prescribers produced the vast majority of DMT prescriptions (10 232/12 628 or $81 \%)$. In contrast, the $100 / 286(35 \%)$ neurologists working at an address with an MS clinic generated only $6749 / 12628$ (53\%) DMT prescriptions. The number of neurologists classified as MS specialists using the geographical criterion is inevitably going to be an over-estimate as non-MS specialists in the same hospital will also be included. However, since more prescriptions were generated by the 37 high-volume prescribers than the $100 \mathrm{MS}$ clinic based neurologists, the prescription-based definition of MS specialists must by necessity include some purely community-based neurologists who do not see patients in one of the provincial MS clinics.

This study is the first to attempt to link DMT prescribing patterns with characteristics of the prescribing neurologist. Prescribing patterns have usually been examined from the MS patient perspective by examining which patient characteristics are associated with receiving a script for DMT. ${ }^{16-22}$ Administrative databases have been used to assess physician prescribing practices for MS patients in Nova Scotia, ${ }^{23}$ Wales, ${ }^{24}$ and Norway. ${ }^{25}$ The Nova Scotian and Welsh studies examined all types of prescriptions while the Norwegian study specifically examined DMT prescribing by neurologists. In that study, prescribing rates obtained from the national Norwegian Prescription Database were compared with the estimated MS prevalence rates in the eight of nineteen counties where this information was available. ${ }^{23}$ The authors found markedly variable rates of DMT utilization, ranging from $19-47 \%$ of patients (average $28 \%$ ), across the country. This study examined DMTs in aggregate and did not examine whether individual DMTs were more commonly prescribed by specific neurologists.

Several limitations of this study merit discussion. First, confidentiality rules precluded MS specialist neurologists (known by name to the authors) from being isolated from the rest of the Ontario neurologists. To circumvent this issue, the neurologists with a primary practice address corresponding to the postal code of one of the MS clinics in the province were used as a proxy for "MS clinic" despite the fact that this group would include other academic subspeciality or generalist neurologists working in the same hospital. Second, the ICES databases do not contain prescription information for Ontario residents whose medication costs are covered by private insurance plans. However, the percentages of the four DMTs obtained from the commercial Brogan database for the year 2009 (available at the aggregate level for the whole province) are comparable to the percentages reported here, suggesting that our results are external valid (data not shown). Provincial confidentiality rules prevent linkage of commercial databases with the ICES data holdings.
While differences in prescribing patterns were noted between specialists and generalists using the prescription-volume dichotomization, no differences were apparent comparing Avonex prescriptions to the other three first-line DMTs. It is important to note that because of the specific nature of the hypothesis-driven questions asked, this analysis cannot examine the degree to which a single "non-Avonex" medication was distributed amongst either the high- or low-volume prescribers. It is likely that individual prescribers do not each prescribe all three non-Avonex DMTs equally; for example one physician may favour Copaxone and another Betaseron. As these prescriptions are being combined in aggregate however, only the cumulative proportion of total prescriptions "non-Avonex" prescriptions were plotted in these Lorenz curves.

One issue we could not explore which could affect neurologist prescribing is the degree of interaction with the pharmaceutical industry. Given that the annual cost of the four first-line DMTs ranges from \$16,000-24,000 CDN/year/patient, there is a lot of revenue to be generated from MS DMT prescriptions. It is possible that prescribing at the individual physician level is influenced by the degree of interactions with industry (eg: visits from pharmaceutical representatives, attendance at sponsored meetings). Such influences could affect the prescribing patterns of neurologists, regardless of specialization or prescription volume. It is impossible to quantify this, however, without access to corporate records or the means to link such information to the ICES data holdings. Individual physician perspectives on the cost-effectiveness or utility of individual DMTs or DMT in general could not be determined from the administrative databases.

The most important finding from this study was how a small number of neurologists prescribed the overwhelming majority of DMTs in Ontario in 2009. This is perhaps not surprising given the increasing sub-specialization of neurological practice and medicine in general. The skew demonstrating that a small number of MS specialists (defined as high-volume prescribers) generate the majority of DMT prescriptions has both positive and negative policy implications. On the one hand, this skew indicates that MS patients currently do have access to MS Clinic and community-based neurologists who have an active interest in MS and are comfortable with DMT prescribing. Conversely, this finding is of significance with respect to future patient care since this means that MS patients who could benefit from DMT will need to have continued access to the relatively small subset of neurologists who prescribe such drugs. The prevalence of MS as a whole is increasing, so more and more patients will be eligible candidates for DMT and need access to MS-specialists. Our study did not examine natalizumab (Tysabri) which was only added to the Ontario drug formulary in the autumn of 2009. The risk of progressive multifocal leukoencephalopathy (PML) with Tysabri can reasonably be assumed to limit its use by nonspecialists. In Ontario, for example, it is only available through the provincial formulary if prescribed from an MS clinic. Fingolimod (Gilenya), which is not yet available on the formulary, also requires a degree of cardiorespiratory and ophthalmological baseline testing (although subsequent monitoring of patients is not onerous) as well as a willingness to prescribe a drug whose long-term safety has not yet been established. It is unlikely that such a medication will be widely 
embraced by the cohort of neurologists who currently do not prescribe the conventional DMTs which are markedly easier to monitor.

A significant concentration of DMT prescribing has implications for future access by MS patients. Since the highvolume prescribing subset of neurologists includes both MS Clinic-based and community neurologists, any limitations on DMT prescribing by community neurologists through the public catastrophic drug plan would only serve to increase the burden on the MS Clinic system and create a potential bottleneck delaying patient care. Future planning for the care of MS patients must therefore ensure that adequate resources are in place for potential DMT-treatment candidates to receive timely access to both MS clinic and community-based MS specialists.

\section{ACKNOWLEDGEMENT}

James Marriott was funded by the Biogen-Idec Canada MS Clinical Fellowship and the Teva Neuroscience Multiple Sclerosis Fellowship. Gustavo Saposnik is supported by the Distinguished Clinician Scientist Award from the Heart and Stroke Foundation.

\section{CONFLICT OF INTEREST}

James Marriott has received research support for MS trials from Biogen Idec, Roche and sanofi-aventis and honoraria from Biogen Idec and EMD Serono. Muhammad Mamdani has received honoraria for serving on advisory boards for Novartis, Roche, and sanofi-aventis. Paul O'Connor has received consulting fees and/or research support for MS trials from Actelion, Bayer, Biogen Idec, BioMS, Cognosci, Daiichi Sankyo, EMD Serono, Genentech, Genmab, Novartis, Roche, sanofi-aventis, Teva Neurosciences and Warburg Pincus.

\section{REFERENCES}

1. Jacobs LD, Cookfair DL, Rudick RA, et al. Intramuscular interferon beta-1a for disease progression in relapsing multiple sclerosis. Ann Neurol. 1996;39(3):285-94.

2. The IFNB Multiple Sclerosis Study Group. Interferon beta-1b is effective in relapsing-remitting multiple sclerosis. I. clinical results of a multicenter, randomized, double-blind, placebocontrolled trial. Neurology. 1993;43(4):655-61.

3. Paty DW, Li DKB. Interferon beta-1b is effective in relapsingremitting multiple sclerosis. II. MRI analysis results of a multicenter, randomized, double-blind, placebo-controlled trial. Neurology. 1993;43(4):662-7.

4. The IFNB Multiple Sclerosis Study Group and the University of British Columbia MS/MRI Analysis Group. Interferon beta-1b in the treatment of multiple sclerosis: final outcome of the randomized controlled trial. Neurology. 1995;45(7):1277-85.

5. PRISMS Study Group. Randomised double-blind placebocontrolled study of interferon beta-1a in relapsing/remitting multiple sclerosis. Lancet. 1998;352(9139):1498-504.

6. Johnson KP, Brooks BR, Cohen JA, et al. Copolymer 1 reduces relapse rate and improves disability in relapsing-remitting multiple sclerosis: results of a phase III multicenter, doubleblind, placebo-controlled trial. Neurology. 1995(7);45:1268-76.
7. Mikol DD, Barkhof F, Chang P, et al. Comparison of subcutaneous interferon beta-1a with glatiramer acetate in patients with relapsing multiple sclerosis (the REbif vs Glatiramer Acetate in Relapsing MS Disease [REGARD] study): a multicentre, randomised, parallel, open-label trial. Lancet Neurol. 2008;7 (10):903-14.

8. O'Connor P, Filippi M, Arnason B, et al. 250 microg or 500 microg interferon beta- $1 \mathrm{~b}$ versus $20 \mathrm{mg}$ glatiramer acetate in relapsingremitting multiple sclerosis: a prospective, randomised, multicentre study. Lancet Neurol. 2009;8(10):889-97.

9. Gajofatto A, Bacchetti P, Grimes B, et al. Switching first-line disease-modifying therapy after failure: impact on the course of relapsing-remitting multiple sclerosis. Mult Scler. 2009;15(1): 50-8.

10. Noyes K, Bajorska A, Chappel A, et al. Cost-effectiveness of disease-modifying therapy for multiple sclerosis: a populationbased study. Neurology. 2011;77(4):355-63 .

11. Gastwirth JL. The Estimation of the Lorenz Curve and Gini Index. Rev Econ Stat. 1972;54(3):306-16.

12. Lee W-C. Characterizing exposure/disease association in human populations using the Lorenz curve and Gini index. Stat Med. 1997:16(7):729-39.

13. Brown MC. Using Gini-style indices to evaluate the spatial patterns of health practitioners: theoretical considerations and an application based on Alberta data. Soc Sci Med. 1994;38(9): 1243-56.

14. Hallas J, Nissen A. Individualized drug utilization statistics. Eur J Clin Pharmacol. 1994;47(4):367-72.

15. Ogwang T. A convenient method of computing the Gini index and its standard error. Oxford Bull Econ Statist. 2000;62(1):123-9.

16. Ozminkowski RJ, Marder WD, Hawkins K, et al. The use of disease-modifying new drugs for multiple sclerosis treatment in private-sector health plans. Clin Ther. 2004;26(8):1341-54.

17. Avasarala JR, O'Donovan CA, Roach SE, et al. Analysis of NAMCS data for multiple sclerosis, 1998-2004. BMC Med. 2007;5:6.

18. Lundy J, Craig BM. The use of disease-modifying agents among multiple sclerosis patients enrolled in medicare from 1995 to 2002 and the impact of medicare part D: analysis of claims data from the medicare current beneficiary survey. Clin Ther. 2006;28 (1):140-5

19. Lo AC, Hadjimichael O, Vollmer TL. Treatment patterns of multiple sclerosis patients: a comparison of veterans and nonveterans using the NARCOMS registry. Mult Scler. 2005;11(1):33-40.

20. Shabas D, Heffner M. Multiple sclerosis management for lowincome minorities. Mult Scler. 2005;11(6):635-40.

21. Iezzoni LI, Ngo LH, Kinkel RP. Working-age persons with multiple sclerosis and access to disease-modifying medications. Mult Scler. 2008;14(1):112-22.

22. Minden SL, Hoaglin DC, Hadden L, et al. Access to and utilization of neurologists by people with multiple sclerosis. Neurology. 2008;70(13 Pt 2):1141-9.

23. Torkildsen $\varnothing$, Grytten N, Myhr KM. Immunomodulatory treatment of multiple sclerosis in Norway. Acta Neurol Scand Suppl. 2007; 187:46-50

24. Tremlett HL, Luscombe DK, Wiles CM. Prescribing for multiple sclerosis patients in general practice: a case-control study. J Clin Pharm Ther. 2001;26(6):437-44.

25. Sketris IS, Brown M, Murray TJ, et al. Drug therapy in multiple sclerosis: a study of Nova Scotia senior citizens. Clin Ther. 1996; 18(2):303-18. 